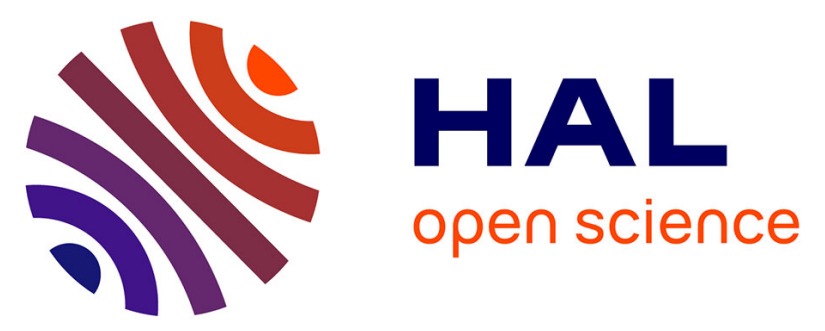

\title{
Analysis of optical filtering in waveguides with a high index modulation using the extended coupled mode theory by hybridization of a matrix method
}

Kamal Ghoumid, Imen Elhechmi, S. Mekaoui, Christian Pieralli, Tijani Gharbi

\section{To cite this version:}

Kamal Ghoumid, Imen Elhechmi, S. Mekaoui, Christian Pieralli, Tijani Gharbi. Analysis of optical filtering in waveguides with a high index modulation using the extended coupled mode theory by hybridization of a matrix method. Optics Communications, 2013, 289, pp.85 - 91. 10.1016/j.optcom.2012.10.008 . hal-00878652

\section{HAL Id: hal-00878652 https://hal.science/hal-00878652}

Submitted on 30 Oct 2013

HAL is a multi-disciplinary open access archive for the deposit and dissemination of scientific research documents, whether they are published or not. The documents may come from teaching and research institutions in France or abroad, or from public or private research centers.
L'archive ouverte pluridisciplinaire HAL, est destinée au dépôt et à la diffusion de documents scientifiques de niveau recherche, publiés ou non, émanant des établissements d'enseignement et de recherche français ou étrangers, des laboratoires publics ou privés. 


\title{
Analysis of optical filtering in waveguides with a high index modulation using the extended coupled mode theory by hybridization of a matrix method
}

\author{
K. GHOUMID ${ }^{1,2, *}$, I. ELHECHMI ${ }^{3}$, S. MEKAOUI ${ }^{4}$, C.Pieralli ${ }^{1}$ and T. \\ $\mathrm{GHARBI}^{3}$ \\ ${ }^{1}$ Institut Femto-ST Département LOPMD, UMR CNRS 6174, Université de \\ Franche-Comté, 16 route de Gray, 25030 Besançon, France. \\ ${ }^{2}$ École Nationale des Sciences Appliquées d'Oujda (ENSAO), Complexe Universitaire, \\ B.P. 669, 60000 Oujda - Morocco. \\ ${ }^{3}$ Nanomedecine Lab, Universite de Franche-Comté, 16 route de Gray, 25030 Besançon, \\ France. \\ ${ }^{4}$ L.C.P.T.S. Télécommunications, Faculté d'Électronique et d'Informatique, USTHB, \\ Alger 16111, Algeria. \\ *kamal.ghoumid@univ-fcomte.fr
}

\begin{abstract}
In this paper the authors present an hybrid approach for the analysis of the optical filtering function in corrugated waveguide filters with a high index modulation. This approach is based on the hybridization of the extended couple mode theory (CMT) with the transfer matrix approach. The authors chose to treat the case of high index corrugation because in this case the theory elaborated before is not rigorously applicable. The proposed approach allows the calculation of the reflection coefficient and the full width at half maximum (FWHM) for any index modulation scheme. The hybridization of both methods mentioned above explains the impact and effects of optogeometric parameters on the reflection coefficient and the bandwidth at midheight. The theoretical results are verified by experimental measurements realized on $\mathrm{Ti}: \mathrm{LiNbO}_{3}$ waveguides with a high index modulation experimentally implemented by engraving using Focused Ion Beam (FIB) process.
\end{abstract}

Keywords: Waveguide index modulation, Optical filtering, Diffraction grating, Coupled mode theory, Matrix method. 


\section{Introduction}

The propagating waves in an optical waveguide which presents an undulation of its refractive index are partially or thoroughly reflected with generating at a time an optical filter which can stop some waves with specific wavelengths ranges. This filter can also be considered as a reflector of some specific wavelength. Then, the produced waves are classified into two main categories such as : a co-propagating wave wave $\xi_{p}^{+}(z)$ that propagates in the direction of the incident propagation vector and another contra-propagating wave $\xi_{p}^{-}(z)$ in the reverse direction $[1,2]$.

The relation between co-propagating and contra-propagating waves is determined by a coupling coefficient $\kappa$ which explicitly describes the energy transfer between both kinds of waves $[2,3]$. Among the various methods and techniques that can treat this phenomenon, we find the well known multilayer process $[4,5,6,7]$, also called the matrix method [8, 9]. It is simply based upon the consideration of a series of $\mathrm{N}$ alternating bi-layers having different refraction indices. This technique is not so powerful because it does not allow the coupling calculation between both different propagating waves and does not take under consideration all of the physical parameters involved in the phenomenon especially the guidance properties of waves.

It should also be noted that many authors were concerned by solving numerically the Maxwell's equations and were also responsible in making their solution answering reliably the treated problem. Note also that some rigorous methods have been formulated in order to calculate spectral properties of a deeply etched short Bragg grating in a comparatively high-contrast optical waveguide $[10,11]$, where a comparison of modeling approaches and verification of computer codes (modal, finite-difference time-domain (FDTD), and grating methods) used by different laboratories had been made. Few differences were revealed; they showed up essentially identical results, and are thus believed to be highly reliable. This fact led to the possibility of accurate 2D numerical modeling of radiation losses of the slab PCs in more depth.

The concept of the coupling modes theory [12], is another more powerful process as compared to the previous one. In a first step, it requires the calculation of the electric field at any space point of the propagating field. The process allows then the calculation of the index corrugation in a second step. In a third step, Maxwell's equations are used to obtain the coupling equations whose solving cannot be possible without acceptable specific approximations. The most difficult step of this theory is that it is conditioned 
by a precise domain where approximations are validated [13]. The various conditions that are required to efficiently satisfy this technique are: (i) index modulation, which in fact is the variation of the refraction index between the guiding layer of the waveguide and its neighbors, must be weak; (ii) The product between the coupling coefficient $\kappa$ and the perturbation length $L$, must be in the order of few unities $[14,15]$.

In this paper, we present a modified model which is based on the solving of Maxwell's equations associated to the transfer matrix approach applied to high amplitude of index modulation in optical waveguides. This model can also be used with weak, medium or with high amplitude of corrugation indices. It implies no approximations and the theoretical predictions have been compared with experimental measurements. This concept can be considered as an extension of the coupled modes theory in the case of using high values of modulation indices. As a main application, we have used this concept to study Bragg Grating (B.G) created by an index perturbation inside a waveguide in order to calculate both the reflection coefficient and the bandwidth at mid-height.

This concept permits to know the influence and effect of the following parameters: (i) The corrugation depth l; (ii) The period of the B.G; (iii) The duty cycle ratio $r$; (iv) Bragg interference order $m$; (v) refraction index $n_{3}$ of the superficial layer. The simultaneous control of these parameters leads the fabrication of filters or reflectors in conformity with wished characteristics. The first aim is to study theoretically the calculation of the coupling coefficient in order to control the different effects both on the reflection coefficient and the bandwidth. Then, we proceeded to the experimental measurements (see setup depicted in section III), in order to compare the theoretical results and measurements of B.G obtained by Focused Ion Beam (FIB) etching of a $\mathrm{Ti}: \mathrm{LiNbO}_{3}$ waveguide. The obtained experimental results are in good accordance with those theoretically predicted [14].

\section{Model description and theory}

\subsection{Model description}

Figure 1 shows a representation of the studied model where an index corrugation is engraved inside a waveguide whose index is $n_{2}$ and width is $d$. Index modulation is characterized by a depth $l$, a period $\Lambda$ and a duty cycle $r=\frac{W}{\Lambda}$. The total number of periods is $N$. The length of corrugation is $L=N . \Lambda$. The B.G represented in figure 1 reflects a frequency band around a 
wavelength so-called Bragg's wavelength, according to the following equation $[16,17,18]$ :

$$
2 n_{\text {eff }} \Lambda=m \lambda_{B}
$$

$n_{\text {eff }}$ is the effective index, $m$ is the Bragg's order and $\Lambda$ the period of the BG.

In this structure, we are only interested by the determination of the coupling coefficient that allows to obtain the values of the reflectivity and the frequency pass band by using Maxwell's equations and mathematical methods especially those involving matrices. For that purpose, we should find out the expression of the electric field in the whole space surrounding the structure and that of the index variation $[5,6]$.

It should be noted that the resolution of this model can not be made by the CMT. Indeed, this theory is in fact a set of general approximations schemes used to describe a complex system in the best simplified manner. From the mathematical point of view, the perturbations theory is considered as a general method that roughly estimates an approximate solution to one mathematical equation depending on an unknown parameter when a particular solution is well-known or derived. This method also consists in finding the most appropriate and approximate solution in terms of series expansions of the powers of the unknown parameter. The CMT is then a powerful tool which is based on Maxwell and perturbations theories. It allows the general behavior description of the perturbed systems. Nevertheless, as it requires a certain number of approximations, it appears necessary to duly verify the validation of these approximations when this theory is applied to specific physical configurations. Therefore, it appears that the CMT is based on the turning wave approximation concept and hence it can be rigorously applicable if and only if the approximation is made strictly valid. In the case of perturbed optical waveguides, this theory is applicable only if the index difference is very small or feeble and inasmuch the variations of the amplitudes co-propagating wave $A(z)$ and contra-propagating $B(z)$ can be considered as constant at wavelength scale. Whereas in the case of high index difference $\Delta n$, nothing can guarantee this approximation to be still valid.

\subsection{Expression of the coupling coefficient $\kappa$}

The coupling coefficient $\kappa$ represents the energy transfer between the two types of waves which are propagated e.g. the co-propagating wave and the contra-propagating one that are generated by the index modulation is 


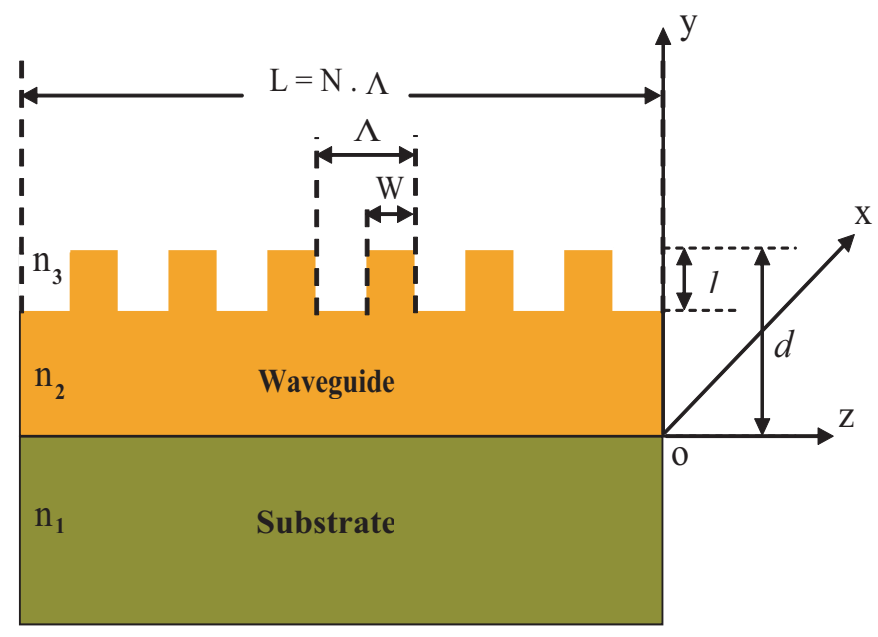

Figure 1: Substrate whose refractive index is $n_{1}$. Waveguide : refractive index $n_{2}$, width $d$, period $\Lambda$, periods number $N$, length $L=N \Lambda$, corrugated index $\left(n_{2}-n_{3}\right)$ and duty cycle (cyclic ratio) $r=\frac{W}{\Lambda}$.

obtained with the help of the above defined variation. Its expression is given by the following equation :

$$
\kappa=\frac{k_{0}^{2}\left(n_{2}^{2}-n_{3}^{2}\right)}{2 \pi m \beta N^{2}} \sin \left(m \frac{\pi W}{\Lambda}\right)\left\{\begin{array}{l}
l_{2}+\frac{\sin \left(2 l_{2} h\right)}{2 h}+\frac{q}{h^{2}}\left[1-\cos \left(2 l_{2} h\right)\right] \\
+\frac{q^{2}}{h^{2}}\left[l_{2}+\frac{\sin \left(2 l_{2} h\right)}{2 h}\right]+\frac{1}{q}\left[1-e^{-2 q l_{1}}\right]
\end{array}\right\}
$$

With

$$
\begin{gathered}
l_{1}=\frac{W}{\Lambda} l \\
l_{2}=\left(1-\frac{W}{\Lambda}\right) l
\end{gathered}
$$

It is obvious from equation (2) that the coupling coefficient depends on several parameters, namely: the depth of corrugation $l$; the duty cycle (cyclic ratio) $r$; the Bragg order $m$; the index variation $\left(n_{2}-n_{3}\right)$.

Based on the coupled modes theory, the expressions of the maximum reflectivity and the bandwidth at mid-height (Nyquist rate) are respectively given by, $[19]$ :

$$
\Re_{\text {Max }}=\tanh ^{2}(\kappa L)
$$




$$
\Delta \lambda=\frac{\lambda_{B}^{2}}{n_{\text {eff }} L} \sqrt{1+\left(\frac{\kappa L}{\pi}\right)^{2}}
$$

However at this level, it is worth noting that equations (5) and (6) cannot be applied in the case that the difference $\left(n_{2}-n_{3}\right)$ is high. In this case, the approximation calculated in equation (5) is not respected. To treat one example like this depicted in figure (1), it is necessary to modify the classical coupled-modes theory by adding to this concept the matrix based process. This process is derived from the description of multilayer dielectric mirrors.

\subsection{Use of matrix based method}

The method consists in writing a matrix $M$ on one period, then obtaining the global matrix $M_{t o t}$ which represents the total corrugation by simply writing $M_{t o t}=M^{N}$ (the same basic motive $M$ is repeated $N$ times), where $N$ is the total number of periods. We can then derive a relation between the fields $E^{+}$and $E^{-}$respectively co-propagating and contra-propagating at space points $z=0$ and $z=L$, in terms of $[20,21]$ :

$$
\left(\begin{array}{c}
E^{+}(z=0) \\
E_{-}(z=0)
\end{array}\right)=[M]^{N}\left(\begin{array}{c}
E^{+}(z=L) \\
E_{-}(z=L)
\end{array}\right)
$$

Where the $M$ matrix elements are given by the followings, [5]:

$$
\left\{\begin{array}{l}
m_{11}=\left[\cosh (\gamma L)+i \frac{\Delta \beta}{\gamma} \sinh (\gamma L)\right] \exp \left(-i \beta_{B} L\right) \\
m_{12}=-\frac{\kappa}{\gamma} \sinh (\gamma L) \exp \left[-i\left(\beta_{B} L+\Phi_{k}\right)\right] \\
m_{21}=\left(m_{1 P}^{1 P}\right)^{*} \\
m_{22}=\left(m_{11}^{1 P}\right)^{*}
\end{array}\right\}
$$

With :

$$
\begin{gathered}
\Delta \beta=\beta-\beta_{B} \\
=\beta-\frac{\pi}{\Lambda} \\
\gamma=\sqrt{\kappa^{2}-\Delta \beta^{2}} \\
\Phi_{k}=\Phi^{k-1}+\frac{2 \pi L^{k}}{\Lambda^{k}} \quad \text { for } \quad 0 \leq k \leq N
\end{gathered}
$$

It can easily find out the value of the reflectivity $\Re$ at a given wavelength $\lambda$. This reflectivity is then defined by :

$$
\Re(\lambda)=\left|\frac{M_{21}}{M_{11}}\right|^{2}
$$




\section{Opto-geometric parameters effects on reflectivity and bandwidth}

This theoretical approach was verified first by simulation to insure that the results are consistent with what physics predicts. In a second step, the simulation was used to define experimental parameters B.G realized on guide $\mathrm{Ti}: \mathrm{LiNbO}_{3}$ whose properties were measured.

\subsection{Effects of corrugation depth $l$ and Bragg's order $m$}

To study the effects of the Bragg order $m$ and depth of corrugation $l$, simulations have been implemented to draw curves representing the coupling coefficient $\kappa$ as a function of the depth of corrugation for different values of the Bragg order $m$ and the reflectivity curve as a function of wavelength $\lambda$. These curves are shown in figures 2 and 3.

This increase of coupling coefficient induces an increase of bandwidth at half maximum $\Delta \lambda$. On the other hand the maximum of reflectivity remains constant $\Re_{\text {Max }}(m=1) \approx \Re_{\text {Max }}(m=3) \approx \Re_{\text {Max }}(m=5)$.

This result is logical and can be explained by the fact that the product $\kappa L$ is constant for these different orders. We have $\kappa(m=1)>\kappa(m=3)>$ $\kappa(m=5)$, whereas this is the exact reverse for the total lengths $L$ of the BG $L(m=1)<L(m=3)<L(m=5)$. Consequently $\kappa(m=1) L(m=1) \approx$ $\kappa(m=3) L(m=3) \approx \kappa(m=5) L(m=5)$.

Concerning the bandwidth, which is inversely proportional to the total length $L$ of the B.G, it decreases from order $m=1$ to order $m=5$.

\subsection{Effect of duty cycle $r$}

The coupling coefficient $\kappa$ also depends on the duty cycle $r=\frac{W}{\Lambda}$ and consequently on the values of reflectivity and bandwidth. This is clearly observed on figure 4 and figure 5 illustrated hereafter. It appears that this effect is periodic with a period $T=1 / m$ and that its maximum value is obtained whenever the duty cycle $r_{i}$ satisfies equation (13) with $0<i<m-1$ :

$$
r_{i}=\frac{1}{2 m}+\frac{i}{m}
$$

For example, if the Bragg order is $m=5$, the maximum values of $\kappa$ are obtained for : $r_{0}=0.1, r_{1}=0.3, r_{2}=0.5, r_{3}=0.7$ and $r_{4}=0.9$ (see figure 5).

Consequently, the coupling coefficient $\kappa$ is equal to zero when $\sin (m \pi r)=0$ that corresponds to the values $r=0.2,0.4,0.6,0.8$ and 1 .

It can be deduced that, for a duty cycle $r=\frac{1}{2}$, the coupling coefficient $\kappa$ is 


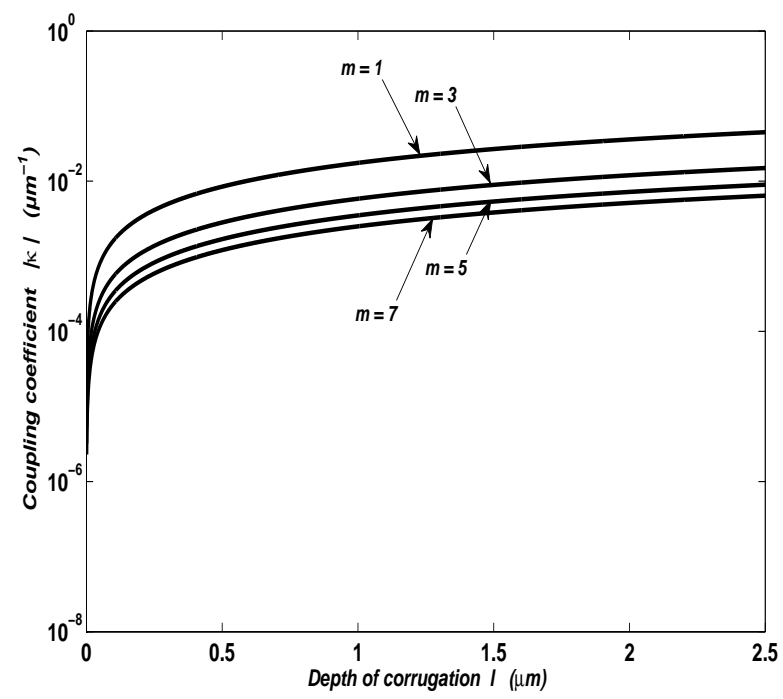

Figure 2: Coupling coefficient $\kappa$ versus the corrugation depth $l$ for different values of Bragg's order $m$. (simulation data: $n_{1}=2.2112, n_{2}=2.2174, n_{3}=1, d=5 \mu m$ and $r=0.5)$.

equal to zero for an even order $m=2,4,6, \ldots$. For a duty cycle $r=\frac{1}{3}$, the coupling coefficient $\kappa$ is equal to zero for orders which are multiples of 3 i.e. $m=3,6,9,12 \ldots$

\subsection{Effect of the refractive index $n_{3}$ of the superficial layer}

In this section, we are interested in studying the effect of the layer whose index is $n_{3}$. It is clear from equation (2), that this layer has an important effect since the difference $\left(n_{2}-n_{3}\right)$ appears in the coupling coefficient expression. We observe from the curves depicted in figure 6 and figure 7 that the coupling coefficient, the reflectivity and the bandwidth or the band pass are decreasing when the index value $n_{3}$ is increasing.

\subsection{Effect of the periods number $N$}

We have implemented and realized simulations in order to study the effect of the periods number $N$. For this purpose, we have represented the variations of the reflectivity $\Re$ versus the wavelength $\lambda$ for different values of $N$. These simulations are illustrated in figure 8. The well-known theoretical result taken in its domain of validity from the coupled modes theory 


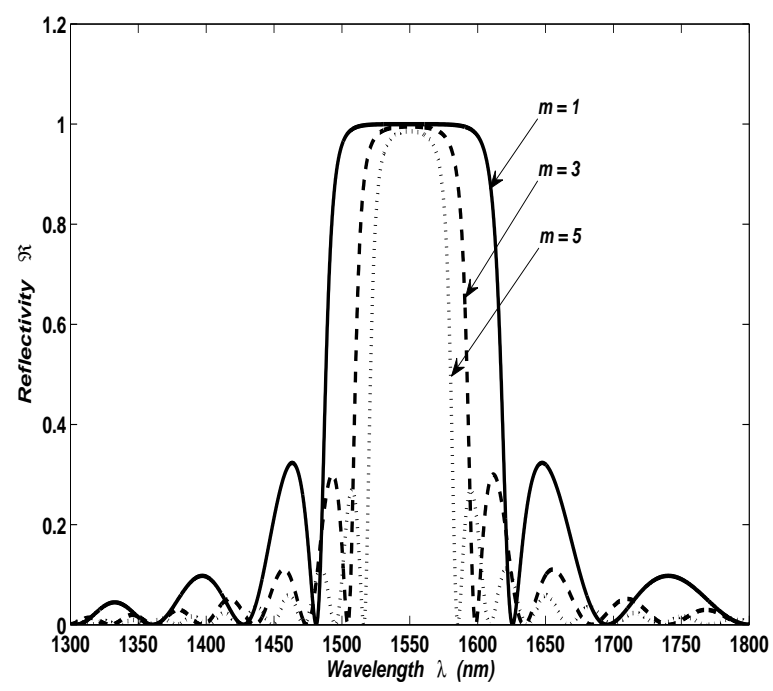

Figure 3: Reflectivity $\Re$ versus wavelength $\lambda$ for different values of the Bragg's order $m$. (simulation data : $n_{1}=2.2112, n_{2}=2.2174, n_{3}=1, d=5 \mu m, r=0.5$ and $N=60$ ).

for the multilayer pattern remains valid and correct in this new extended approach. Furthermore, it remains applicable to modulations characterized by high index values. In addition, this result asserts that reflectivity becomes considerably important when the periods number $N$ increases. Nevertheless, this increase in reflectivity is done to the detriment of the mid- height bandwidth which in contrast diminishes when the periods number $N$ increases.

\section{Model Validation and authentication from the experimental re- sults}

\subsection{B.G fabrication methods}

Among the most well known methods for the fabrication of BGs, we can find the following methods such as $[22,23,24,25]$ : the photo-refractive method, the Reactive Ion Etching (RIE), or the Depth Reactive Ion Etching (DRIE), laser ablation, protonic exchange, holographic interferometer methods using for example a Sagnac Interferometer. The majority of these methods requires long and heavy steps of fabrication like: mask design; lithography; resin development; engraving and so on.

We adopted a technique based upon the method named Focused Ion Beam 


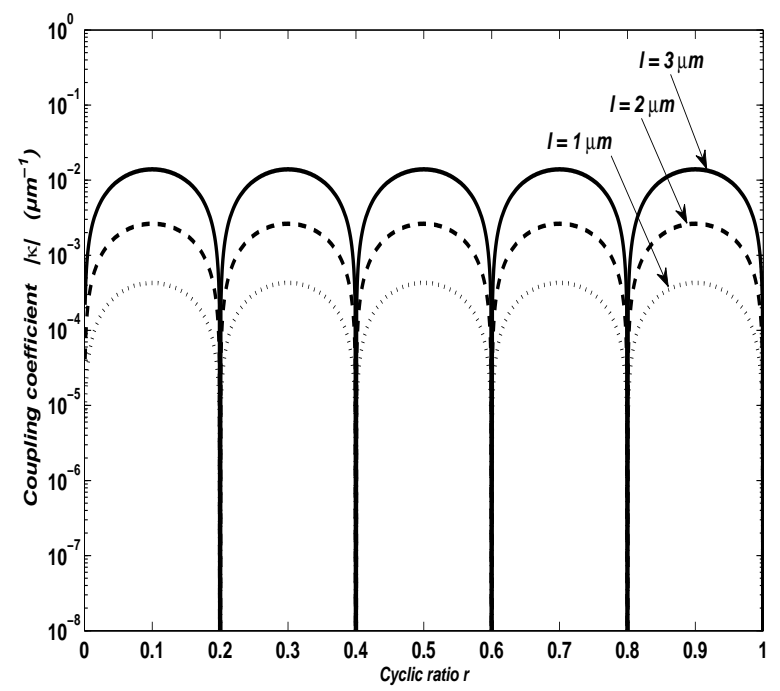

Figure 4: The coupling coefficient $\kappa$ versus the duty cycle $r$ for different values of engraved depth $l$.(simulation data : $n_{1}=2.2112, n_{2}=2.2174, n_{3}=1, d=5 \mu m$ and $m=5$ ).

(FIB) for fabricating our B.G This technique drastically reduces the number of fabrication steps. Engraving is directly made in this case without involving the use of a mask. Its main advantage is to offer the possibility to design very tiny B.G with dimensions lower than $50 \mathrm{~nm}$. This level of performance is difficult to obtain with the previous cited methods[26, 27].

\subsection{Waveguides and $B G$ fabrication}

The wave guide is fabricated by standard diffusion at a temperature of $T=1020^{\circ} \mathrm{C}$ during 10 hours, a titanium ribbon of width $7 \mu \mathrm{m}$ and of a thickness of $80 \mathrm{~nm}$ deposited by sputtering on a sample of lithium niobate $\left(\mathrm{LiNbO}_{3}\right)$ of type : $\mathrm{X}$-cut, $\mathrm{Z}$ propagation. The waveguide is single mode for a central wavelength of about $1.55 \mu \mathrm{m}$ and TM polarization. The dimensions of this wave guide are: Width $W_{x}=4,32 \mu \mathrm{m}$ and Depth $W_{y}=3,61 \mu \mathrm{m}$ (These dimensions are measured at $1 / e$ relatively to the maximum).

Once the waveguide is elaborated, the next step consists in engraving the B.G. This step is realized by the mean of the FIB technique. This technique of engraving is of an ionic type, it is realized step by step, period by period. The B.G obtained by FIB technique is presented in [14, 19]. This B.G. has a period of $\Lambda=1.75 \mu \mathrm{m}$ and a width $L=N . \Lambda=20 \times 1.75=35 \mu \mathrm{m}$. The 


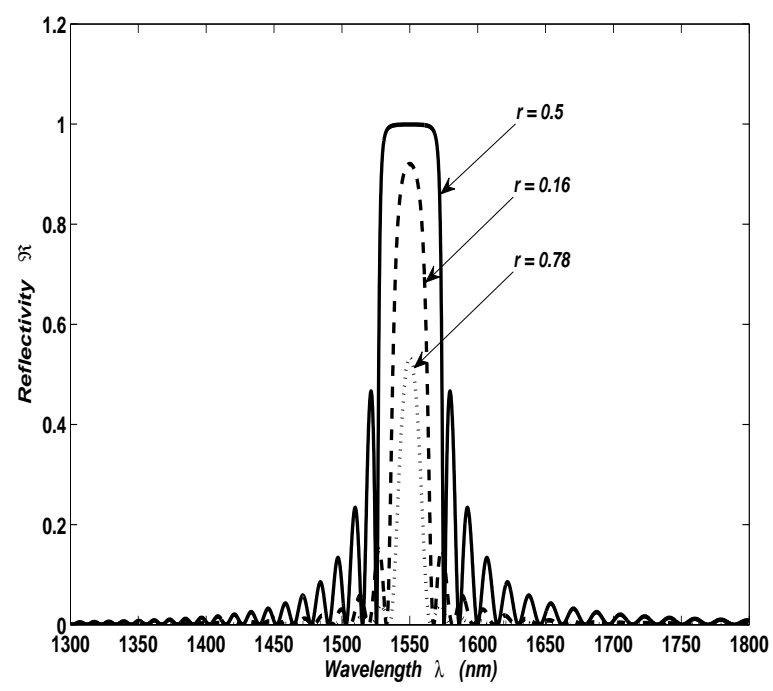

Figure 5: Reflectivity $\Re$ versus wavelength $\lambda$ for different values of the duty cycle $r$. (simulation data : $n_{1}=2.2112, n_{2}=2.2174, n_{3}=1, d=5 \mu m, r=0.5, m=5$ and $N=65)$.

shape of the holes obtained by FIB engraving is illustrated in $[14,28]$. We have to pay attention to the side wall profiles (left and right) obtained have an inclination in respect to the vertical. We have noticed that because of this slope, the cyclic ratio $r$ does not remain constant; it increases with the depth of etching.

\subsection{Reflectivity Measurements}

The experimental setup that allows us to measure the reflectivity in terms of the wavelength is described in reference $[14,18]$. It is composed by a continuous spectrum source $[850-1750] \mathrm{nm}$, a set of focusing components to inject light in waveguide, a polarizer to select the electric-field orientation, a beam splitter to separate the input light and the reflected light and an Optical Spectrum Analyzer (OSA).

To compare theoretical properties and experimental results, the variation of three parameters has been considered : The periods number $N$, the Bragg order $m$ and the engraved depths $l$. Figure 9 represents the reflectivity curve versus the wavelength $\lambda$ for different values of $N$. We can observe that reflectivity increases when the periods number $N$ increases 


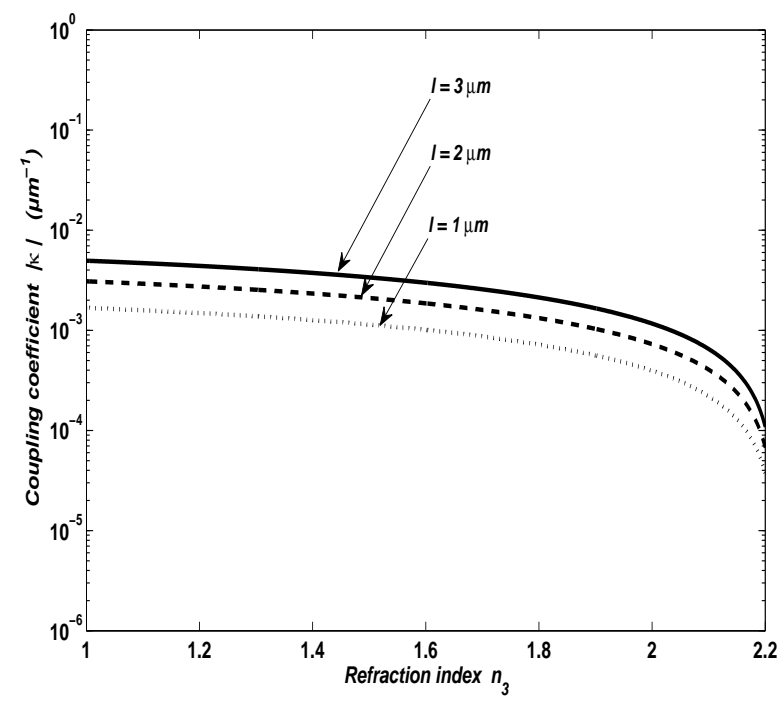

Figure 6: The coupling coefficient $\kappa$ versus refractive index $n_{3}$ for different values of engraved depth $l$. (simulation data : $n_{1}=2.2112, n_{2}=2.2174, d=5 \mu m, m=7$ and $r=0.5)$.

$\Re(N=80) \approx 96 \%>\Re(N=40) \approx 75 \%>\Re(N=30) \approx 15 \%$, and we specifically get whereas the bandwidth decreases at half maximum $\Delta \lambda(N=$ $80)<\Delta \lambda(N=40)<\Delta \lambda(N=30)$.

Figure 10 represents the reflectivity versus the wavelength for different values of $l$. It indicates that reflectivity increases when the engraved depth $l$ increases $\Re(l=2.8 \mu \mathrm{m}) \approx 97 \%>\Re(l=2 \mu \mathrm{m}) \approx 90 \%>\Re(l=1.7 \mu \mathrm{m}) \approx$ $70 \%$, and bandwidth at mid-height decreases when $l$ increases $\Delta \lambda(l=2.8 \mu \mathrm{m})<$ $\Delta \lambda(l=2 \mu m)<\Delta \lambda(l=1.7 \mu m)$.

Figure 11 depicts the reflectivity curve versus the wavelength for different values of $m$. What is observed in this case is that reflectivity is approximately constant even if when Bragg's order $m$ increases $\Re(m=3) \approx$ $\Re(m=5) \approx \Re(m=7) \approx 97 \%$, and bandwidth decreases when $m$ decreases $\Delta \lambda(m=7)<\Delta \lambda(m=5)<\Delta \lambda(m=3)$.

It is worth noting that these experimental results are shown in the curves of Figures 9, 10 and 11 confirm perfectly and are in good agreement with the theoretical curves found above.

Finally, it is important to observe that random parasites peaks appear on the reflectivity curves which are probably due to the roughness of the holes. 


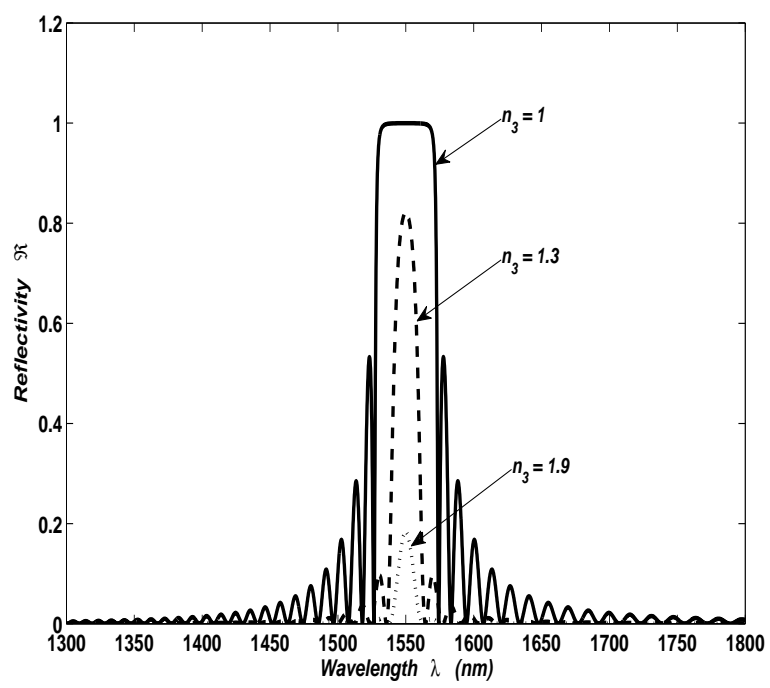

Figure 7: Reflectivity $\Re$ versus wavelength $\lambda$ for different values of the refractive index $n_{3}$. (simulation data : $n_{1}=2.2112, n_{2}=2.2174, d=5 \mu m, m=7, r=0.5$ and $N=60$ ).

\section{Conclusion}

The main difficulty in manufacturing of integrated optical component is due to the multiplicity of technological operations. Each of then is difficult and it is imperative that they must be perfectly successful. The number of parameters used in these operations is very high. In addition, there is a strong interaction between these parameters. An experimental and systematic study of the influence of these parameters to optimize performances of the integrated device is impossible to achieve due to the number of combinations.

A realistic theoretical description of physical phenomena in integrated optical that permits to write computing software simulating propagation of light is of an imperious necessity. Today, the best and complete description of propagation in waveguide is obtained by use of the Coupled Mode Theory. But, it appears, in very specific cases, that this theory does not give expected results. Especially, when the index variation is high in a corrugated waveguide, the approximations of variation method cannot be validated. To overcome this difficulty that appears in the case of B.Gs fabricated by FIB technique, a hybridization of the coupled mode theory has been developed. It consists 


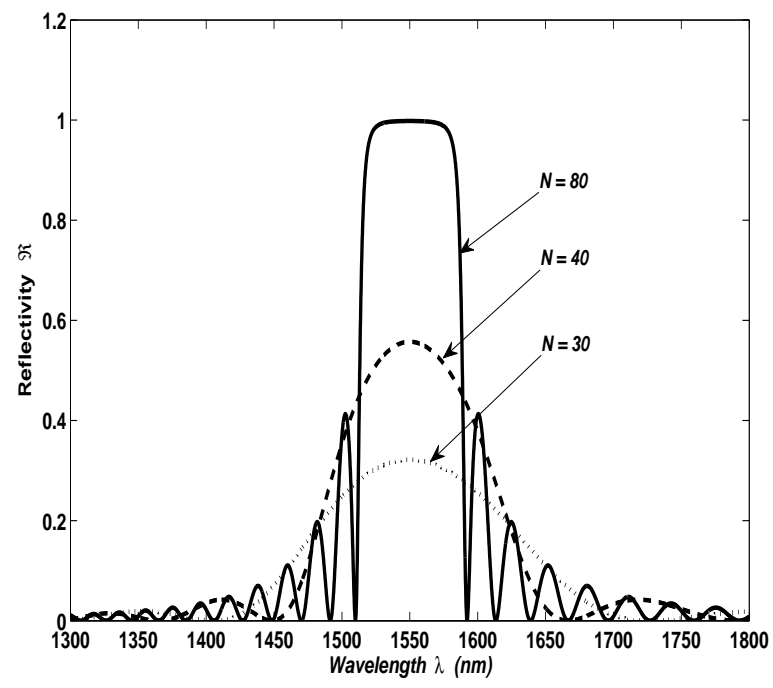

Figure 8: Reflectivity $\Re$ versus wavelength $\lambda$ for different values of the periods number $N$. (simulation data : $n_{1}=2.2112, n_{2}=2.2174, n_{3}=1, d=5 \mu m, m=5$ and $r=0.5$ ).

in evaluating the coupling coefficient on a period of B.G. and to repeat the result with the matrix method commonly used to describe multilayer model. This model applied to a B.G indicates how several parameters can act and be adjusted (corrugation depth $l$; cyclic ratio $r$; Bragg's order $m$; periods number $N$; refractive index $n_{3}$ of superstrate) for mastering the coupling between contra-propagating and co-propagating waves. It permits to control coupling coefficient, reflectivity and bandwidth.

Experimental measurements are in good accordance with predicted values supplied by this theoretical model. Thus, we have obtained a set of experimental parameters that allow to design reflectors whose reflectivity coefficient is close to $100 \%$ associated with different bandwidths at mid-height depending on the needs. 


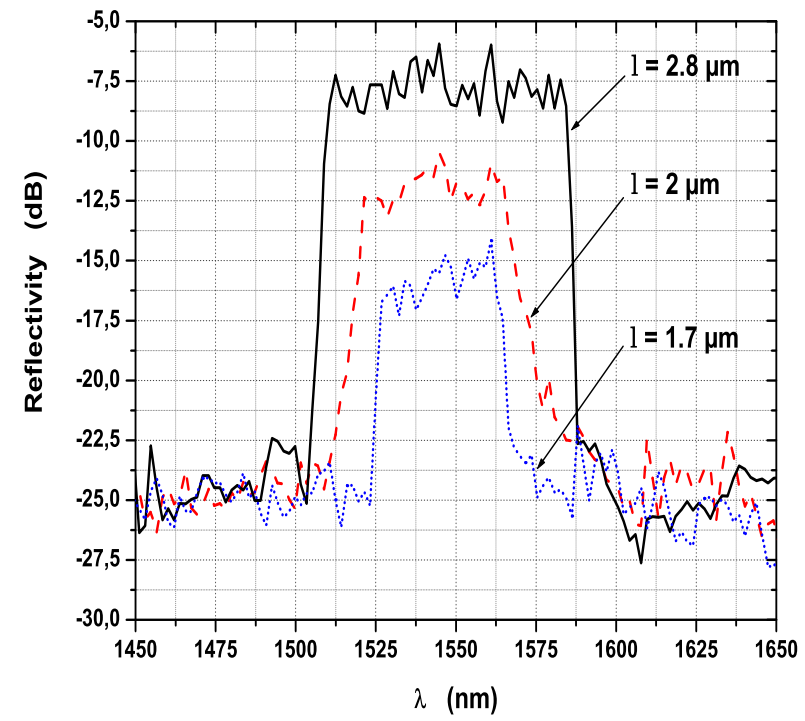

Figure 9: Experimental reflectivity versus wavelengths for different depth corrugations $l$.

\section{References}

[1] A. Yariv, 'coupled-mode theory for guided-wave optics', IEEE. J. Quantum Electronics, 9, (1973) 919 - 933.

[2] A. Yariv, P. Yeh, 'Optical Waves in Crystal: Propagation and control of Laser Radiation',, New York,, New York, 1984.

[3] T. Suhara, H. Nishihara, 'integrated optics components and devices using periodic structures',, IEEE J. Quantum Electronics, 22, (1986,) 845 $-867,$.

[4] M. McCall, 'on the application of coupled mode theory for modeling fiber bragg gratings',, IEEE. J. Lightwave Tech. 2, (2000) 236 - 242.

[5] W. Streifer, D. Scifres, R. Burnham, 'coupling coefficients for distributed feedback single-and double-heterostructure diode lasers', , IEEE J. Quantum Electronics, 11, (1975) $867-873$. 


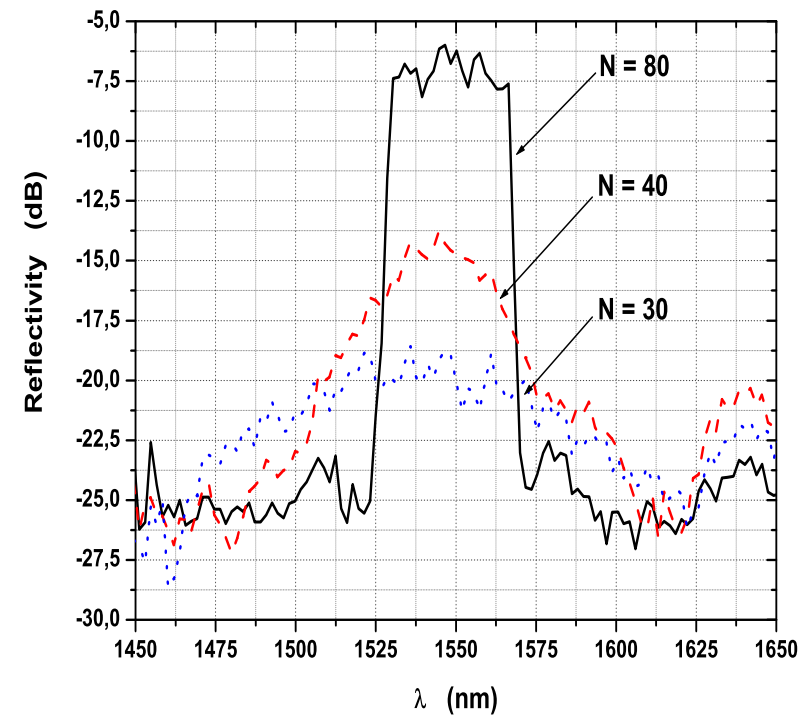

Figure 10: Experimental reflectivity versus wavelengths for different period's number $N$.

[6] R. Burnham, D. Scifres, W.Streifer, 'single-heterostructure distributedfeed-back gaas-diode lasers',, IEEE. J. Quantum Electronics, 11, (1975) $439-449$.

[7] A. Hardy, W. Streifer, 'coupled mode theory of parallel waveguides',, IEEE. J. Lighwave Tech. 3, (1985) 1135 -1146.

[8] J. Hong, W. Huang, T. Makino, 'on the transfer matrix method for distributed-feedback waveguide devices',, IEEE. J. Lightwave Technology, 10, (1992) $1860-1868$.

[9] B. Kim, E. Garmire, 'comparison between the matrix method and the coupled-wave method in the analysis of bragg reflector structures', , J. Opt. Soc. 9, (1992) $132-136$.

[10] A. Yariv, M. Nakamura, 'periodic structure for integrated optics', IEEE J. Quantum Electron, 13, (1977,) 233 - 251,.

[11] R. P. P. B. R. B. R. D. R. R. S. G. K. J. P. C. P. L. J.-P. H. J. Ctyroky, S. Helfert, R. M. D. L. Rue, bragg waveguide grating as a $1 \mathrm{~d}$ photonic 


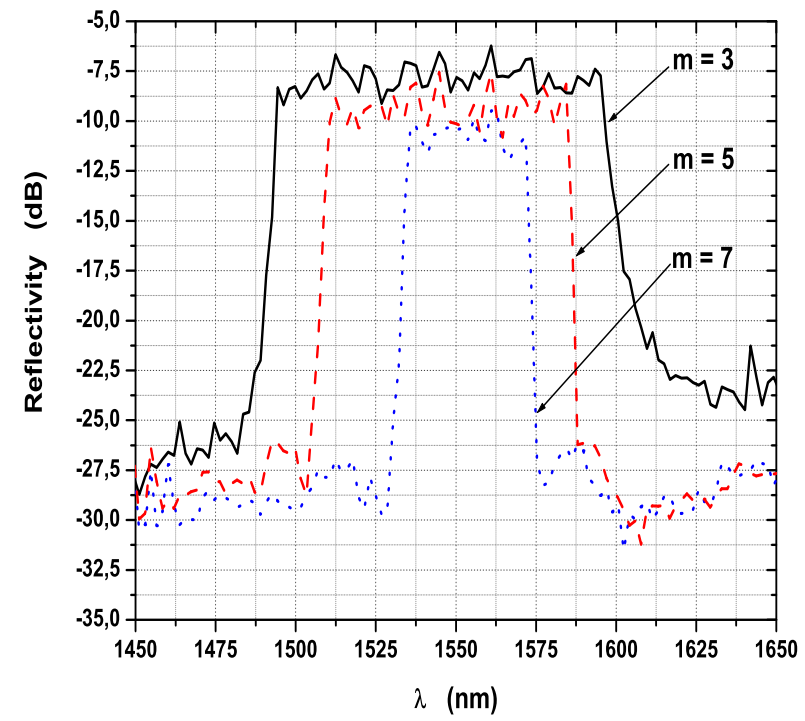

Figure 11: Experimental reflectivity versus wavelengths for different Bragg's order $m$.

band gap structure: Cost 268 modelling task', Optics and Quantum Electronics, 34, (2002,) $455-470$.

[12] A. Sharma, P.-K. Mishra, A.-K. Ghatak, 'single-mode optical waveguide and directional couplers with rectangular cross section : A simple and accurate method of analysis',, IEEE. J. Lightwave Tech. 6, (1988,) 1119 $-1126,$.

[13] N. Matuschek, F. Krtner, U. Keller, 'exact coupled-mode theorys for multilayer interference coatings with arbitrary strong index modulations',, IEEE J.Quantum Electron, 33, (1997,) 295 -302,.

[14] K. Ghoumid, R. Ferrière, B.-E. Benkelfat, B. Guizal, T. Gharbi, 'optical performance of bragg gratings fabricated in $\mathrm{Ti}_{\mathrm{LiNbO}}$ waveguides by focused ion beam milling', , IEEE, J. Lightwave Tech, 28 (2010) 3488 3493, .

[15] K. Ghoumid, R. Ferrière, B.-E. Benkelfat, S. Mekaoui, C. Benmouhoub, T. Gharbi, 'technological implementation fabry-pérot cavity 
in Ti: $\mathrm{LiNbO}_{3}$ waveguide by fib', IEEE, Phot. Tech. Letters 24 (2012) $231-233$.

[16] K. Hill, T. Fujii, D. Johnson, B. Kawasaki, 'photosensitivity in optical fiber waveguides: Application to reflection filter fabrication',, Appl. Phys. Let, 32, 32, (1978,) 647-649,.

[17] T. Erdogan, 'fiber grating spectra',, IEEE. J. Lighwave Tech. 15, (1997,) $1277-1294$.

[18] R. Ferrière, B.-E. Benkelfat, J. Dudley, K. Ghoumid, 'bragg miroir inscription on $\mathrm{LiNbO}_{3}$ waveguides by index microstructuration',, J. Appl. $45,(2006) 1-$,9 .

[19] K. Ghoumid, R. Ferrière, B.-E. Benkelfat, G.Ulliac, R. Salut, J.-Y. Rauch, T. Gharbi, 'effect of depth etching on bragg grating reflector realized by focused ion beam in $\mathrm{Ti}_{\text {: }} \mathrm{LiNbO}_{3}$ waveguides',, J. SPIE, 7386 (2), (2009,) $1-6$,.

[20] M. Yamada, K. Sakuda, 'analysis of almost-periodic distributed feedback slab waveguide via a fundamental matrix approach',, Appl. Opt., 26, (1987,) $3476-3478$,.

[21] M. Prabhugoud, K. Peters, 'modified transfer matrix formulation for bragg grating strain sensors',, IEEE. J. Lightwave Tech. 22, (2004,) 2302 -2309 .

[22] C. Becker, A. Oesselke, A. Pape, W. Sohler, H. Suche, 'integrated optical Ti:Er:LiNbO ${ }_{3}$ distibuted bragg reflector laser with fixed photorefractive grating',, Opt. Letters, 23, (1998,) $1194-1196$,.

[23] B. Das, R.Ricken, W. Sohler, 'integrated optical distributed feedback laser with Ti:Fe: $\mathrm{LiNbO}_{3}$ waveguide',, Appl.Phys. Letters, 82, (2003,) $1515-1517$,

[24] J. Sochtig, 'Ti: $\mathrm{LiNbO}_{3}$ stripe waveguide bragg reflector gratings',, J. Elect. Letters, 24, (1988,) $844-845$,.

[25] Y. Sidorin, A. Cheng, 'integration of bragg gratings on $\mathrm{LiNbO}_{3}$ channel waveguides using laser ablation',, Electr. Letters, 37, (2001,) 312 - 314,. 
[26] L. Pierno, M. Dispenza, A. Secchi, A. Fiorello, V. Foglietti, 'a lithium niobate electro-optic tunable bragg filter fabricated by electron beam lithography',, J. Opt. A: Pure Appl. Opt. 10, (2008,) 1 -6,.

[27] S. Yin, 'fabrication of high-aspect-ratio submicron-to-nanometer range microstructures in $\mathrm{LiNbO}_{3}$ for the next generation of integrated optoelectronic devices by focused ion beams (fib)',, Microwave and optical technology letters. 22, $(1999) 396-398$, .

[28] K. Ghoumid, B.-E. Benkelfat, R. Ferrière, T. Gharbi, 'wavelengthselective $\mathrm{Ti}_{\mathrm{i}} \mathrm{LiNbO}_{3}$ multiple y-branch coupler based on focused ion beam milled bragg reflectors', IEEE, J. Light. Tech. 29 (2011) 3536 $-3541$. 